\title{
Optimized Beamforming and Backhaul Compression for Uplink MIMO Cloud Radio Access Networks
}

\author{
Yuhan Zhou and Wei Yu \\ Department of Electrical and Computer Engineering \\ University of Toronto, Toronto, Ontario, Canada \\ Email: $\{$ yzhou,weiyu $\}$ ece.utoronto.ca
}

\begin{abstract}
This paper studies the optimization of transmit beamforming and backhaul compression strategies for the uplink of cloud radio access networks (C-RAN), in which multi-antenna user terminals communicate with a cloud-computing based central processor (CP) through multi-antenna base-stations (BSs) serving as relay nodes. The BSs perform compress-and-forward strategy to quantize the received signals and send the quantization bits to the CP via capacity-limited backhaul links for decoding. In contrast to the previous works on the uplink C-RAN, which mostly focus on the backhaul compression strategies only, this paper proposes the joint optimization of the transmit beamformers and the quantization noise covariance matrices at the BSs for maximizing the benefit brought by the C-RAN architecture. A weighted sumrate maximization problem is formulated under the user power and backhaul capacity constraints. A novel weighted minimummean-square-error successive convex approximation (WMMSESCA) algorithm is developed for finding a local optimum solution to the problem. This paper further proposes a low-complexity approximation scheme consisting of beamformers matching to the strongest channel vectors at the user side along with per-antenna scalar quantizers with uniform quantization noise levels across the antennas at each BS. This simple separate design strategy is derived by exploring the structure of the optimal solution to the sum-rate maximization problem under successive interference cancellation (SIC) while assuming high signal-to-quantizationnoise ratio (SQNR). Simulation results show that with optimized beamforming and backhaul compression, $\mathrm{C}$-RAN can significantly improve the overall performance of wireless cellular networks. With SIC, the proposed separate design performs very close to the optimized joint design in the SQNR regime of practical interest.
\end{abstract}

\section{INTRODUCTION}

To meet the exponential increasing data demand in wireless communication driven by smartphones, tablets, and video streaming, modern cellular systems are moving towards heterogenous networks consisting of base-stations (BSs) deployed with high density, covering progressively smaller areas. As a consequence, inter-cell interference management becomes a challenging problem. Cloud radio access network (C-RAN) is a promising network architecture for dealing with this interference problem. In a C-RAN architecture, the encoding/decoding functionalities of the BSs are migrated to a cloudcomputing based central processor $(\mathrm{CP})$. By taking advantage of high backhaul links between the $\mathrm{CP}$ and the BSs, C-RAN allows coordination and joint signal processing across multiple cells, and the possibility for inter-cell interference cancellation, which has the potential to significantly improve the overall throughput of the wireless cellular network [1].
One of the main problems arising in the practical implementation of C-RAN is how to optimally utilize the backhaul when the backhaul link capacities are finite. Substantial research works have made progress towards this direction, either from information theoretic point of view [2]-[4] or algorithm design point of view [5], [6]. In [7], a virtual multiple access channel (VMAC) scheme, which is a two-stage compress-and-forward strategy based on successive decoding, is proposed for the single-input single-output (SISO) C-RAN architecture. It is shown that the VMAC scheme can achieve the sum capacity of C-RAN to within a constant gap under a sum backhaul capacity constraint.

This paper studies the linear transceiver and backhaul compression design in the VMAC scheme for the uplink multipleinput-multiple-output (MIMO) C-RAN model as shown in Fig. 1. As a generalization of [7] which considers the SISO case only, this paper considers the MIMO case where both the users and the BSs are equipped with multiple antennas. The main difference between the SISO case and the MIMO case is the impact of transmit optimization at the user terminals. In the SISO case, since most of the intra-cluster interference has been nulled by multicell decoding, it is near optimal for the users to transmit with their maximum powers. In the MIMO case, the users are capable of doing transmit beamforming, so the optimal transmit beamforming design is more involved. This is especially so when the backhaul links in C-RAN have limited capacities.

The backhaul compression problem for the uplink C-RAN model has been considered extensively in the literature. Various algorithms such as the gradient projection method [5], the robust backhaul compression approach [6], and the alternating convex optimization approach [7] are developed for maximizing the (weighted) sum rate under the bachaul constraints. All of these algorithms focus only on the optimization of quantization noise covariance matrices across the BSs, while keeping the transmit beamformers fixed. This paper goes one step further by considering the joint transmit beamformer and quantization noise covariance matrix optimization problem. Accounting for both the transmit beamforming and the quantization design problem together in the optimization framework is nontrivial because the two are coupled through the backhaul constraints. To tackle this problem, this paper proposes a weighted minimum-mean-square-error successive convex approximation (WMMSE-SCA) algorithm for efficiently finding 


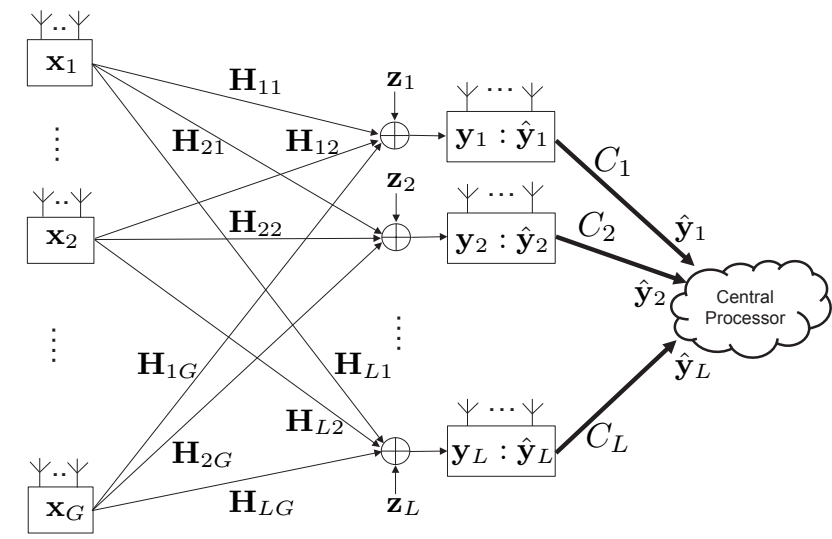

Fig. 1. Uplink of a cloud radio-access network with capacity-limited backhaul

a local optimum solution to the weighted sum rate maximization problem. Moreover, a simple approximation scheme consisting of transmit beamformers matching to the strongest channel signal vector and per-antenna scalar quantizers with uniform quantization noise levels across the antennas at each $\mathrm{BS}$ is also developed for the sum-rate maximization problem when the signal-to-quantization-noise ratio (SQNR) is high and when successive interference cancelation (SIC) is applied at the receiver. This low-complexity scheme designs the transmit beamforming and the backhaul compression separately. $\mathrm{Nu}$ merical simulations show that with SIC implemented at the receiver the proposed separate design is near optimal in the SQNR regime of practical interest.

\section{SyStem Model AND PROBLEM Formulation}

We consider the uplink of a C-RAN, where $G$ mobile users communicate with a $\mathrm{CP}$ through $L \mathrm{BSs}$, as shown in Fig. 1. The BSs are connected to the CP via noiseless backhaul links of finite capacities $C_{i}$. Each user terminal is equipped with $M$ antennas, and each BS is equipped with $N$ antennas. In this paper, we assume that the user scheduling is fixed, and perfect channel state information (CSI) is made available to all the $\mathrm{BSs}$ and to the CP.

The VMAC scheme with single-user compression is applied in the C-RAN system, where the $i$ th BS quantizes the received signal using single-user compression, then forwards the compressed bits to the $\mathrm{CP}$ for decoding. By single-user compression, we mean that the compression process only involves a conventional vector quantizer, and it does not utilize the correlation between the received signals across the BSs. At the CP side, the quantization codewords are first decoded, then the user messages are decoded sequentially. Define $\mathbf{H}_{i k}$ as the $N \times M$ complex channel matrix between the $k$ th user and the $i$ th BS, the channel matrix from user $k$ to all the BSs is given as the $N L \times M$ matrix, i.e., $\mathbf{H}_{k}=\left[\mathbf{H}_{1 k}^{T}, \mathbf{H}_{2 k}^{T}, \ldots, \mathbf{H}_{L k}^{T}\right]^{T}$. It is assumed that each user intends to transmit $d$ parallel data streams to the CP. Let $\mathbf{V}_{k} \in \mathbb{C}^{M \times d}$ denote the transmit beamfomer that user $k$ utilizes to transmit signal $\mathbf{s}_{k} \in \mathbb{C}^{d \times 1}$ to the central receiver. Then the transmit signal at user $k$

is given by $\mathbf{x}_{k}=\mathbf{V}_{k} \mathbf{s}_{k}$ with $\mathbb{E}\left[\mathbf{s}_{k} \mathbf{s}_{k}^{H}\right]=\mathbf{I}$. The transmit beamformers are subjected to per-user power constraints, i.e., $\operatorname{Tr}\left(\mathbf{V}_{k} \mathbf{V}_{k}^{H}\right) \leq P_{k}$ for $k=1,2, \ldots, G$. The received signal at BS $i, \mathbf{y}_{i}$, can be expressed as

$$
\mathbf{y}_{i}=\sum_{k=1}^{G} \mathbf{H}_{i k} \mathbf{V}_{k} \mathbf{s}_{k}+\mathbf{z}_{i} \quad \text { for } \quad i=1,2, \ldots, L,
$$

where $\mathbf{s}_{k} \sim \mathcal{C N}(0, \mathbf{I})$ is the intended signal vector for user $k$, and $\mathbf{z}_{i} \sim \mathcal{C N}\left(0, \sigma_{i}^{2} \mathbf{I}\right)$ represents the additive white Gaussian noise at BS $i$. Based on rate-distortion theory, the quantized received signal $\hat{\mathbf{y}}_{i}$ for the $i$ th $\mathrm{BS}$ is given by

$$
\hat{\mathbf{y}}_{i}=\mathbf{y}_{i}+\mathbf{q}_{i}
$$

where $\mathbf{q}_{i} \sim \mathcal{C} \mathcal{N}\left(0, K_{Q_{i}}\right)$ represents the additive Gaussian quantization noise.

Following the results in [7], [8], assuming that the linear minimum-mean-squared-error (MMSE) receiver is applied at the $\mathrm{CP}$, the transmission rate $R_{k}$ for user $k$ for the VMAC scheme is given by

$$
R_{k}=\log \left|\mathbf{I}+\mathbf{V}_{k}^{H} \mathbf{H}_{k}^{H} \mathbf{J}_{k}^{-1} \mathbf{H}_{k} \mathbf{V}_{k}\right|
$$

where

$$
\mathbf{J}_{k}=\mathbf{J}_{k}^{\mathrm{LE}}=\sum_{j \neq k}^{G} \mathbf{H}_{j} \mathbf{V}_{j} \mathbf{V}_{j}^{H} \mathbf{H}_{j}^{H}+K_{z}+K_{Q},
$$

with $K_{z}=\operatorname{diag}\left(\sigma_{i}^{2} \mathbf{I}\right)$ and $K_{Q}=\operatorname{diag}\left(K_{Q_{i}}\right)$. To achieve higher throughput, the SIC scheme can also be applied. In this case, the matrix $\mathbf{J}_{k}^{\mathrm{LE}}$ is replaced by $\mathbf{J}_{k}^{\mathrm{SIC}}$ expressed as

$$
\mathbf{J}_{k}=\mathbf{J}_{k}^{\mathrm{SIC}}=\sum_{j>k}^{G} \mathbf{H}_{j} \mathbf{V}_{j} \mathbf{V}_{j}^{H} \mathbf{H}_{j}^{H}+K_{z}+K_{Q} .
$$

with $K_{z}=\operatorname{diag}\left(\sigma_{i}^{2} \mathbf{I}\right)$ and $K_{Q}=\operatorname{diag}\left(K_{Q_{i}}\right)$. The compression rates at the BSs should also satisfy the backhaul link capacity constraints. Based on the vector quantization theory, the backhaul constraints can be expressed as follows

$$
\log \frac{\left|\sum_{k=1}^{G} \mathbf{H}_{i k} \mathbf{V}_{k} \mathbf{V}_{k}^{H} \mathbf{H}_{i k}^{H}+\sigma_{i}^{2} \mathbf{I}+K_{Q_{i}}\right|}{\left|K_{Q_{i}}\right|} \leq C_{i}
$$

for $i=1,2, \ldots, L$, where $\mathbf{H}_{i k}$ is the complex channel gain matrix between $k$ th user and $i$ th $\mathrm{BS}$.

To characterize the tradeoff between the achievable rates for the users and the system resources, we formulate the following weighted sum-rate maximization problem:

$$
\begin{array}{cl}
\max _{\mathbf{V}_{k}, K_{Q_{i}}} & \sum_{k=1}^{G} \alpha_{k} \log \left|\mathbf{I}+\mathbf{V}_{k}^{H} \mathbf{H}_{k}^{H} \mathbf{J}_{k}^{-1} \mathbf{H}_{k} \mathbf{V}_{k}\right| \\
\text { s.t. } & \mathbf{J}_{k}=\mathbf{J}_{k}^{\mathrm{LE}} \quad \text { or } \quad \mathbf{J}_{k}=\mathbf{J}_{k}^{\mathrm{SIC}} \\
& \log \frac{\left|\sum_{k=1}^{G} \mathbf{H}_{i k} \mathbf{V}_{k} \mathbf{V}_{k}^{H} \mathbf{H}_{i k}^{H}+\sigma_{i}^{2} \mathbf{I}+K_{Q_{i}}\right|}{\left|K_{Q_{i}}\right|} \leq C_{i}, \\
& K_{Q_{i}} \succeq \mathbf{0}, \quad \text { for } i=1,2, \ldots, L, \\
& \operatorname{Tr}\left(\mathbf{V}_{k} \mathbf{V}_{k}^{H}\right) \leq P_{k}, \quad \text { for } k=1,2, \ldots, G,
\end{array}
$$


where $\alpha_{k}$ 's are the weights representing the priorities associated with the mobile users.

Due to the non-convexity of both the objective function and the backhaul capacity constraints in problem (6), finding the global optimum solution of (6) is challenging. We point out that the present formulation (6) can be easily extended to a more general case where the user scheduling strategy is also included. More specifically, one can consider a weighted sum rate over all the users in the network, where the beamformers for the users are set to be the zero vector if they are not scheduled. In this paper, we focuses on the active uses only and assume that the user scheduling is done prior to solving problem (6).

\section{Proposed SOlutions}

\section{A. The WMMSE-SCA Algorithm}

This section presents a novel algorithm which is able to find a stationary point of the problem (6). The main difficulties in solving (6) come from the fact that the objective function and backhaul capacity constraints are both nonconvex functions with respect to the optimization variables. Inspired by the recent work on the WMMSE approach for beamforming design [9], [10], we first reformulate the objective function in problem (6) as a convex function with respect to the MMSE-matrix given by the user's target signal $s_{k}$ and decoded signal $\hat{s}_{k}$ assuming that the MMSE receiver is used. We then linearize the convex objective function and the compression rate expressions in the backhaul constraints of (6) to obtain a convex approximation of the original problem. Finally we successively approximate the optimal solution by optimizing this convex approximation. The idea of convex approximation roots from modern optimization techniques including block successive minimization method and minorize-maximization algorithm, which have been previously applied for solving related problems in wireless communications [11], [12].

Before presenting the proposed algorithm, we first state the following lemma, which is a direct consequence of concavity of the $\log |\cdot|$ function.

Lemma 1: For positive definite Hermitian matrices $\Omega, \Sigma \in$ $\mathbb{C}^{N \times N}$,

$$
\log |\Omega| \leq \log |\Sigma|+\operatorname{Tr}\left(\Sigma^{-1} \Omega\right)-N
$$

with equality if and only if $\Omega=\Sigma$.

By applying Lemma 1 to the first log-determinant term in the backhaul constraint (5) and by setting

$$
\Omega=\sum_{k=1}^{G} \mathbf{H}_{i k} \mathbf{V}_{k} \mathbf{V}_{k}^{H} \mathbf{H}_{i k}^{H}+\sigma_{i}^{2} \mathbf{I}+K_{Q_{i}}
$$

we can approximate the backhaul constraint (5) with the following convex constraint:

$$
\begin{array}{r}
\log \left|\Sigma_{i}\right|+\operatorname{Tr}\left(\Sigma_{i}^{-1}\left(\sum_{k=1}^{G} \mathbf{H}_{i k} \mathbf{V}_{k} \mathbf{V}_{k}^{H} \mathbf{H}_{i k}^{H}+\sigma_{i}^{2} \mathbf{I}+K_{Q_{i}}\right)\right) \\
-\log \left|K_{Q_{i}}\right| \leq C_{i}+N \quad(8)
\end{array}
$$

for $i=1,2, \ldots, L$. It is not hard to see that the original backhaul constraint (5) is always feasible when the convex constraint (8) is feasible. The two constraints are equivalent when

$$
\Sigma_{i}^{*}=\sum_{k=1}^{G} \mathbf{H}_{i k} \mathbf{V}_{k} \mathbf{V}_{k}^{H} \mathbf{H}_{i k}^{H}+\sigma_{i}^{2} \mathbf{I}+K_{Q_{i}} .
$$

Now we approximate the objective function in (6) using the WMMSE approximation. Let $\mathbf{U}_{k} \in \mathbb{C}^{N L \times d}$ be the linear receiver applied at the $\mathrm{CP}$ for recovering $s_{k}$. The transmission rate $R_{k}$ in (2) can be expressed as the following [9] [10],

$$
R_{k}=\max _{\mathbf{U}_{k}} \log \left|\mathbf{E}_{k}^{-1}\right|
$$

where

$$
\begin{aligned}
\mathbf{E}_{k}=(\mathbf{I}- & \left.\mathbf{U}_{k}^{H} \mathbf{H}_{k} \mathbf{V}_{k}\right)\left(\mathbf{I}-\mathbf{U}_{k}^{H} \mathbf{H}_{k} \mathbf{V}_{k}\right)^{H} \\
& +\mathbf{U}_{k}^{H}\left(\sum_{j \neq k}^{G} \mathbf{H}_{j} \mathbf{V}_{j} \mathbf{V}_{j}^{H} \mathbf{H}_{j}^{H}+K_{z}+K_{Q}\right) \mathbf{U}_{k} .
\end{aligned}
$$

By applying Lemma 1 again, we rewrite rate expression (10) as

$$
R_{k}=\max _{\mathbf{W}_{k}, \mathbf{U}_{k}}\left(\log \left|\mathbf{W}_{k}\right|-\operatorname{Tr}\left(\mathbf{W}_{k} \mathbf{E}_{k}\right)+d\right)
$$

where $\mathbf{W}_{k}$ is the weight matrix introduced by the WMMSE method. The optimal $\mathbf{W}_{k}$ is given by

$$
\mathbf{W}_{k}^{*}=\mathbf{E}_{k}^{-1}=\mathbf{I}+\left(\mathbf{U}_{k}^{*}\right)^{H} \mathbf{H}_{k} \mathbf{V}_{k} .
$$

where $\mathbf{U}_{k}^{*}$ is the MMSE receive beamformer given by

$$
\mathbf{U}_{k}^{*}=\left(\sum_{j \neq k} \mathbf{H}_{j} \mathbf{V}_{j} \mathbf{V}_{j}^{H} \mathbf{H}_{j}^{H}+K_{z}+K_{Q}\right)^{-1} \mathbf{H}_{k} \mathbf{V}_{k} .
$$

Using (11) and (8) to replace the objective function and the backhaul constraints in problem (6), we reformulate the weighted sum-rate maximization problem as follows

$$
\begin{array}{cl}
\max _{\substack{\mathbf{V}_{k}, K_{Q_{i}}, \mathbf{U}_{k}, \mathbf{W}_{k}, \Sigma_{i}}} & \sum_{k=1}^{G} \alpha_{k}\left(\log \left|\mathbf{W}_{k}\right|-\operatorname{Tr}\left(\mathbf{W}_{k} \mathbf{E}_{k}\right)\right) \\
& +\rho \sum_{i=1}^{L}\left\|\Sigma_{i}-\Omega_{i}\right\|_{F}^{2} \\
\text { s.t. } & \log \left|\Sigma_{i}\right|+\operatorname{Tr}\left(\Sigma_{i}^{-1} \Omega_{i}\right)-\log \left|K_{Q_{i}}\right| \leq C_{i}^{\prime}, \\
& K_{Q_{i}} \succeq \mathbf{0}, \text { for } i=1,2, \ldots, L, \\
& \operatorname{Tr}\left(\mathbf{V}_{k} \mathbf{V}_{k}^{H}\right) \leq P_{k}, \text { for } k=1,2, \ldots, G,
\end{array}
$$

where $\rho$ is some positive constant ${ }^{1}, C_{i}^{\prime}=C_{i}+N$, and $\Omega_{i}=\sum_{k=1}^{G} \mathbf{H}_{i k} \mathbf{V}_{k} \mathbf{V}_{k}^{H} \mathbf{H}_{i k}^{H}+\sigma_{i}^{2} \mathbf{I}+K_{Q_{i}}$. Note that the last term in the objective function which involves a summation of Frobenius norms is a quadratic regularization item. It makes problem (14) a strongly convex optimization with respect to each optimization variable.

It is easy to verify that problem (14) is convex with respect to any one of the optimization variables when the other

\footnotetext{
${ }^{1}$ In the simulation part of this paper, $\rho$ is set to be 1000 .
} 
optimization variables are fixed. Specifically, when the other variables are fixed, the optimal values of $\Sigma_{i}, \mathbf{W}_{k}$, and $\mathbf{U}_{k}$ are given by equations (9), (12), and (13) respectively. When $\Sigma_{i}$, $\mathbf{U}_{k}$, and $\mathbf{W}_{k}$ are fixed, the optimal values of $\mathbf{V}_{k}$ and $K_{Q_{i}}$ are the solution of the following optimization problem:

$$
\begin{array}{cl}
\min _{\mathbf{V}_{k}, K_{Q_{i}}} & \sum_{k=1}^{G} \alpha_{k} \operatorname{Tr}\left(\mathbf{W}_{k} \mathbf{E}_{k}\right)+\rho \sum_{i=1}^{L}\left\|\Omega_{i}-\Sigma_{i}\right\|_{F} \\
\text { s.t. } & \operatorname{Tr}\left(\Sigma_{i}^{-1} \Omega_{i}\right)-\log \left|K_{Q_{i}}\right| \leq C_{i}^{\prime}-\log \left|\Sigma_{i}\right|, \\
& K_{Q_{i}} \succeq \mathbf{0}, \quad \text { for } i=1,2, \ldots, L, \\
& \operatorname{Tr}\left(\mathbf{V}_{k} \mathbf{V}_{k}^{H}\right) \leq P_{k}, \quad \text { for } k=1,2, \ldots, G,
\end{array}
$$

The above problem is convex over $\mathbf{V}_{k}$ and $K_{Q_{i}}$, and can be solved efficiently with polynomial complexity. We summarize the proposed WMMSE-SCA algorithm as below:

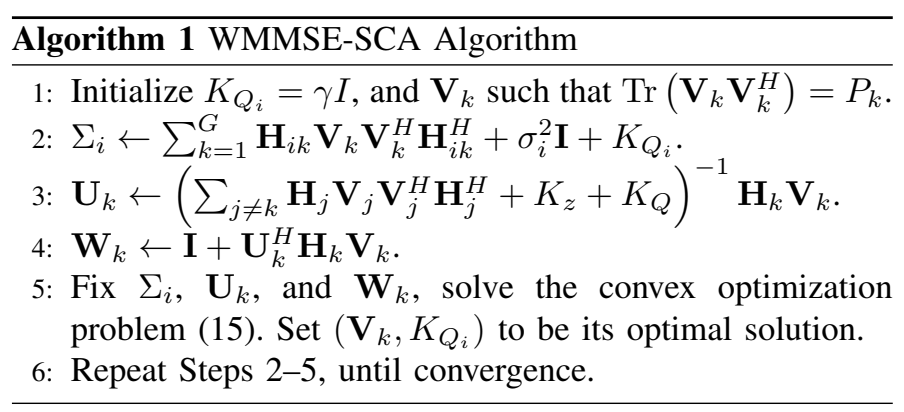

The WMMSE-SCA algorithm yields a nondecreasing sequence of objective values for problem (6). So the algorithm is guaranteed to converge. Moreover, it converges to a stationary point of the optimization problem. The convergence result is stated in Theorem 2. Due to space limit, we omit the proof.

Theorem 2: From any initial point $\left(\mathbf{V}_{k}^{(0)}, K_{Q_{i}}^{(0)}\right)$, the limit point $\left(\mathbf{V}_{k}^{*}, K_{Q_{i}}^{*}\right)$ generated by the WMMSE-SCA algorithm is a stationary point of the weighted sum-rate maximization problem (6).

\section{B. Low-Complexity Beamforming and Quantization Noise De- sign Under High SQNR}

Although locally optimal transmit beamformers and quantization noise covariance matrices can be found using the WMMSE-SCA algorithm for any fixed user schedule, user priority, and channel condition, the implementation of WMMSESCA in practice can still be computationally intensive, especially when the channels are fast varying or when the scheduled users in the time-frequency slots change frequently. In this section, we aim at deriving near optimal transmit beamformers and quantization noise covariance matrices in the high SQNR regime. The main result of this section is that a simple separate design which involves transmit beamformers matched to the strongest channel singular vectors at the user side and scalar quantizers with uniform quantization noise levels across the antennas at each BS is approximately optimal for maximizing the sum rate if an appropriate set of users are scheduled. This leads to an efficient way for transmit beamforming and backhaul compression design in the practical C-RAN systems.
The proposed approximate approach is derived by assuming that SIC is implemented at the central receiver. Let $\mathbf{H}$ denote the channel matrix between the users and the BSs and $\mathbf{G}_{i}$ denote the channel matrix between the users and the $i$ th BS (which is assumed to be full rank). Denote $K_{x_{j}}=\mathbf{V}_{j} \mathbf{V}_{j}^{H}$ and $K_{X}=\operatorname{diag}\left(K_{x_{j}}\right)$. The sum-rate maximization problem can be formulated as follows,

$$
\begin{array}{cl}
\max _{K_{x_{j}}, K_{Q_{i}}} & \log \frac{\left|\mathbf{H} K_{X} \mathbf{H}^{H}+K_{z}+K_{Q}\right|}{\left|K_{z}+K_{Q}\right|} \\
\text { s.t. } & \log \frac{\left|\mathbf{G}_{i} K_{X} \mathbf{G}_{i}^{H}+\sigma_{i}^{2} \mathbf{I}+K_{Q_{i}}\right|}{\left|K_{Q_{i}}\right|} \leq C_{i} \\
& K_{Q_{i}} \succeq \mathbf{0}, \quad \text { for } i=1,2, \ldots, L, \\
& \operatorname{Tr}\left(K_{x_{j}}\right) \leq P_{j}, \quad \text { for } j=1,2, \ldots, G,
\end{array}
$$

where $K_{z}=\operatorname{diag}\left(\sigma_{i}^{2} \mathbf{I}\right)$, and $K_{Q}=\operatorname{diag}\left(K_{Q_{i}}\right)$.

First, we provide a justification that the optimal quantization noise levels should be set as uniform across the antennas at each BS under high SQNR. Towards this end, we derive the Karush-Kuhn-Tucker (KKT) condition for the optimization problem (16) under the high SQNR assumption. To obtain the KKT condition, form the Lagrangian

$$
\begin{aligned}
& L\left(K_{x_{j}}, K_{Q_{i}}, \lambda_{i}, \mu_{j}\right)=\log \left|\mathbf{H} K_{X} \mathbf{H}^{H}+K_{z}+K_{Q}\right| \\
& -\log \left|K_{z}+K_{Q}\right|-\sum_{i=1}^{L} \lambda_{i} \log \left|\mathbf{G}_{i} K_{X} \mathbf{G}_{i}^{H}+\sigma_{i}^{2} \mathbf{I}+K_{Q_{i}}\right| \\
& +\sum_{i=1}^{L} \lambda_{i} \log \left|K_{Q_{i}}\right|-\sum_{j=1}^{G} \mu_{j} \operatorname{Tr}\left(K_{x_{k}}\right)
\end{aligned}
$$

where $\lambda_{i}$ is the Lagrangian dual variable associated with the $i$ th backhaul constraint, and $\mu_{j}$ is Lagrangian multiplier for the $j$ th transmit power constraint.

Setting $\partial L / \partial K_{Q_{i}}$ to zero, we obtain the following optimality condition

$$
\begin{gathered}
\mathbf{F}_{i}\left(\mathbf{H} K_{X} \mathbf{H}^{H}+K_{z}+K_{Q}\right)^{-1} \mathbf{F}_{i}^{T}-\left(\sigma_{i}^{2} \mathbf{I}+K_{Q_{i}}\right)^{-1} \\
-\lambda_{i}\left(\mathbf{G}_{i} K_{X} \mathbf{G}_{i}^{H}+\sigma_{i}^{2} \mathbf{I}+K_{Q_{i}}\right)^{-1}+\lambda_{i} K_{Q_{i}}^{-1}=\mathbf{0}
\end{gathered}
$$

where $\mathbf{F}_{i}=\left[\mathbf{0}, \ldots, \mathbf{0}, \mathbf{I}_{N}, \mathbf{0}, \ldots, \mathbf{0}\right]$ with only the $i$ th $N \times N$ block being nonzero. It is easy to verify that the above optimality condition can only be satisfied if $0 \leq \lambda_{i}<1$. Furthermore, if the overall system is to operate at reasonably high spectral efficiency, where the received signal-tonoise ratios (SNRs) are high and the backhaul capacities are large, we must have $\mathbf{H} K_{X} \mathbf{H}^{H}+K_{z}+K_{Q} \gg K_{z}+$ $K_{Q}$ and $\mathbf{G}_{i} K_{X} \mathbf{G}_{i}^{H}+\sigma_{i}^{2} \mathbf{I}+K_{Q_{i}} \gg K_{Q_{i}}$. Under this high SQNR condition, $\left(\mathbf{H} K_{X} \mathbf{H}^{H}+K_{z}+K_{Q}\right)^{-1} \approx \mathbf{0}$ and $\left(\mathbf{G}_{i} K_{X} \mathbf{G}_{i}^{H}+\sigma_{i}^{2} \mathbf{I}+K_{Q_{i}}\right)^{-1} \approx \mathbf{0}$. Then the optimality condition becomes $\left(\sigma_{i}^{2} \mathbf{I}+K_{Q_{i}}\right)^{-1} \approx \lambda_{i} K_{Q_{i}}^{-1}$, i.e.,

$$
K_{Q_{i}} \approx \frac{\lambda_{i}}{1-\lambda_{i}} \sigma_{i}^{2} \mathbf{I}
$$

where $\lambda_{i} \in[0,1)$ is chosen to satisfy the backhaul capacity constraints. The above result implies that uniform quantization noise levels across the antennas at each BS are optimal at 
high SQNR, (although the quantization noise level may differ from BS to BS depending on the background noise levels and the backhaul constraints.) Note that this line of reasoning is very similar to the corresponding condition for the SISO case derived in [7].

Next, we consider the optimal transmit beamforming and power allocation under high SQNR. Intuitively speaking, for maximizing the sum rate each user should align its signaling direction with the strongest eigen-mode of the effective channel and allocate power along this direction in a "waterfilling" fashion. Towards this end, we need to whiten the combined quantization and background noise and interference, then diagonalize the resulting channel to find its eigenmodes, and iteratively perform the water-filling process among the users [13]. As we see from (19), at high SQNR, the optimal quantization noise covariance matrices are diagonal with entries proportional to the background noise levels. Further, if we choose $d=\min \{M, N L / G\}$, i.e., if we let the total number of user data streams be equal to the number of degrees of freedom in the system, then multiuser interference would be reasonably contained. In this case it is reasonable for each user to transmit with maximum power and allocate the transmit power uniformly among the transmit beamformers.

Based on the above intuition on beamforming design, we propose a low-complexity beamformer design for C-RAN to simply choose the transmit beamformers for each user to match to its transmit channel. Specifically, for user $k$, first perform singular value decomposition on $\mathbf{H}_{k}$ as $\mathbf{H}_{k}=\Phi_{k} \Gamma_{k} \Psi_{k}^{H}$. Then choose the transmit beamformer

$$
\mathbf{V}_{k}=\sqrt{\frac{P_{k}}{d}} \Psi_{k}(1: d)
$$

where $\Psi_{k}(1: d)$ is a matrix formed by $d$ columns of $\Psi_{k}$ which corresponding to the $d$ largest singular values of $\mathbf{H}_{k}$. This channel-matching beamformer together with per-antenna scalar quantizer provide us a low-complexity approximation scheme, which separately designs the transmit beamforming and the backhaul compression. Simulation results in the later section show that with SIC such a separate design performs very close to the optimized joint design in the SQNR regime of practical interest.

Define

$$
C\left(\beta_{i}\right)=\log \frac{\left|\sum_{k=1}^{G} \mathbf{H}_{i k} \mathbf{V}_{k} \mathbf{V}_{k}^{H} \mathbf{H}_{i k}^{H}+\sigma_{i}^{2} \mathbf{I}+\beta_{i} \mathbf{I}\right|}{\left|\beta_{i} \mathbf{I}\right|} .
$$

We summarize the approximation scheme as below

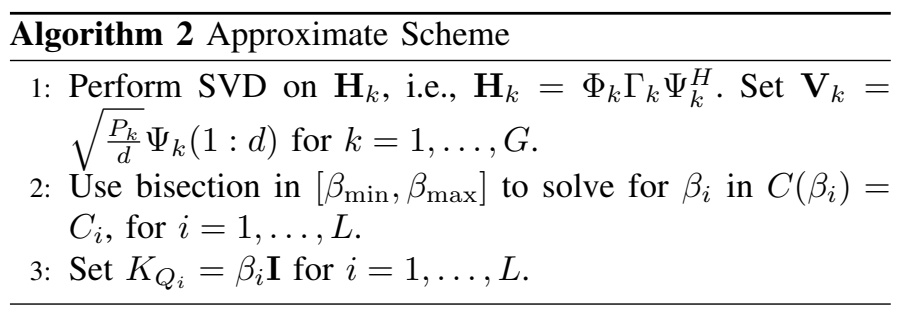

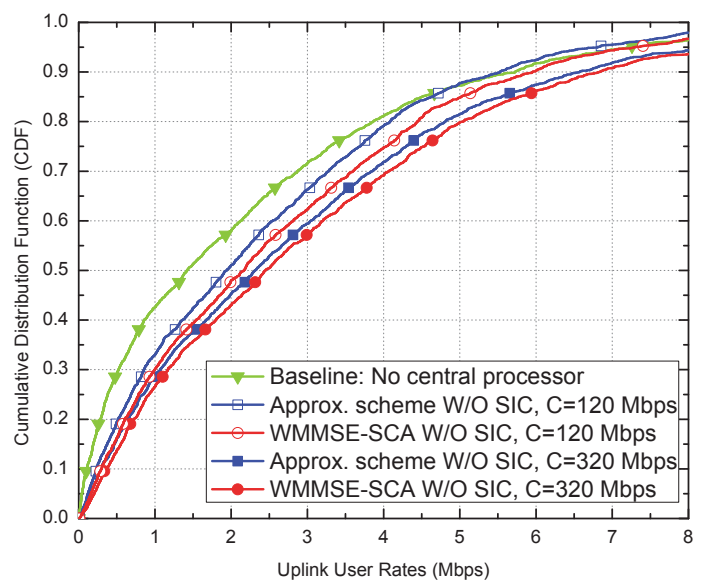

Fig. 2. Cumulative distribution of user rates without SIC

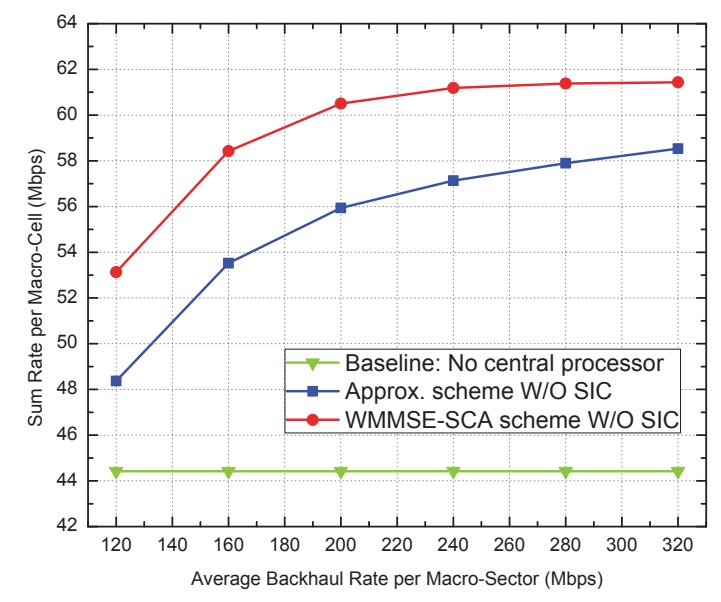

Fig. 3. Per-cell sum rate vs. average per-sector backhaul capacity of the proposed schemes without SIC.

\section{Simulation Results}

In this section, the performances of the proposed WMMSESCA and approximation schemes are evaluated in a 19-cell 3sector/cell wireless network setup with central 7 cells (i.e., 21 sectors) forming a cooperating cluster. The users are randomly located and associated with the strongest BS. Round-robin user scheduling is used on a per-sector basis. Standard cellular network parameters are used in simulation: the noise power spectral density is $-162 \mathrm{dBm} / \mathrm{Hz}$; flat fading channel vectors are chosen according to a distance-dependent path-loss model $128.1+37.6 \log _{10}(d)$, where $d$ is the distance in $\mathrm{km}$, with $8 \mathrm{~dB}$ lognormal shadowing and a Rayleigh component. The distance between neighboring BSs is $500 \mathrm{~m}$. An antenna gain of $14 \mathrm{dBi}$ is assumed. The maximum transmit power constraint for each user is $23 \mathrm{dBm}$, and channel bandwidth is $10 \mathrm{MHz}$. Each BS is equipped with $N=2$ antennas; each user is equipped with $M=2$ antennas; $G=N L$ users are scheduled simultaneously in each time slot, where each user sends one data stream (i.e., $d=1$ ) to the CP.

Fig. 2 and Fig. 3 compare the performance of the baseline 


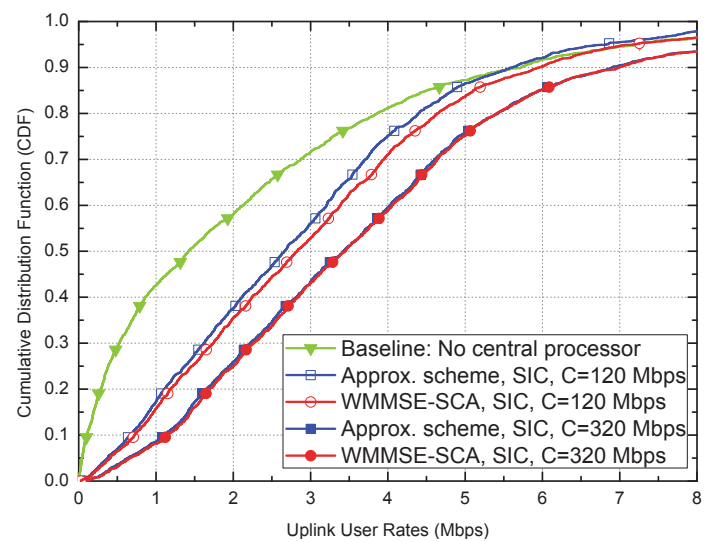

Fig. 4. Cumulative distribution of user rates with SIC.

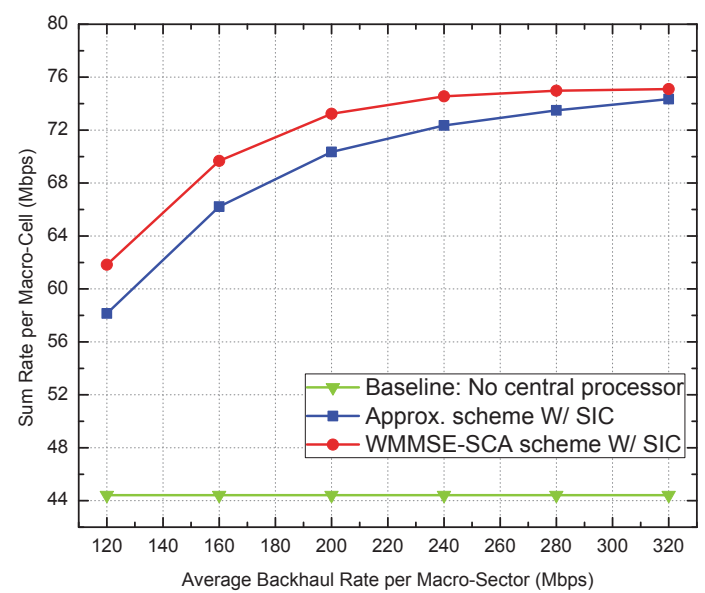

Fig. 5. Per-cell sum rate vs. average per-cell backhaul capacity of the proposed scheme with SIC.

system with the proposed beamforming and backhaul compression schemes implemented without SIC at the receiver. It is shown that both of the WMMSE-SCA and approximation schemes significantly outperform the baseline scheme without multicell processing. The figure also shows that WMMSE-SCA scheme always outperforms the approximation scheme when SIC is not implemented. As the backhaul capacity increases, the performance gap between these two schemes becomes smaller. However, as shown in Fig. 3 such a gap does not vanish even in the high SQNR regime, which demonstrates that the joint design is strictly better than the separate design when the $\mathrm{CP}$ does not implement SIC.

Fig. 4 and Fig. 5 show the performance of the proposed schemes with SIC implemented at the receiver. As compared to the case without SIC, the proposed WMMSE-SCA and approximation schemes can achieve more gain on the overall performance with SIC applied. The performance improvement is more significant for the users with low rate (e.g. 10percentile users), as shown in 4. The figures also show that the approximation scheme performs very close to the WMMSESCA scheme, especially when backhaul capacity is large. As shown in Fig. 5, when the backhaul capacity goes to infinity, the gap between the two proposed scheme vanishes. This demonstrates the approximate optimality for separate design of transmit beamforming and backhaul compression in the high SQNR regime.

\section{CONCLUSION}

This paper studies transmit beamforming and backhaul compression design for an uplink MIMO C-RAN system. We propose a novel WMMSE-SCA algorithm to efficiently optimize the transmit beamformer and quantization noise covariance matrix for maximizing the weighted sum rate. Further, it is shown that with SIC at the receiver, channel-matching beamforming with uniform power allocation at the users together with scalar quantization with uniform quantization noise levels across the antennas at each BS is near optimal for maximizing the sum rate when SQNR is high. This leads to a lowcomplexity algorithm, with separate transmit beamforming and backhaul compression design. Simulation results show that with the optimized transmit beamformer and quantization noise covariance matrix, the C-RAN architecture can significantly improve the performance of wireless cellular networks, and majority of the gain can be achieved by the proposed lowcomplexity scheme.

\section{REFERENCES}

[1] D. Gesbert, S. Hanly, H. Huang, S. Shamai, O. Simeone, and W. Yu, "Multi-cell MIMO cooperative networks: A new look at interference," IEEE J. Sel. Areas Commun., vol. 28, no. 9, pp. 1380-1408, Dec. 2010.

[2] A. Sanderovich, S. Shamai, Y. Steinberg, and G. Kramer, "Communication via decentralized processing," IEEE Trans. Inf. Theory, vol. 54, no. 7, pp. 3008-3023, Jul. 2008.

[3] A. Sanderovich, O. Somekh, H. V. Poor, and S. Shamai, "Uplink macro diversity of limited backhaul cellular network," IEEE Trans. Inf. Theory, vol. 55, no. 8, pp. 3457-3478, Aug. 2009.

[4] L. Zhou and W. Yu, "Uplink multicell processing with limited backhaul via per-base-station successive interference cancellation," IEEE J. Sel. Areas Commun., vol. 31, no. 10, pp. 1981-1993, Oct. 2013.

[5] A. del Coso and S. Simoens, "Distributed compression for MIMO coordinated networks with a backhaul constraint," IEEE Trans. Wireless Commun., vol. 8, no. 9, pp. 4698-4709, Sep. 2009.

[6] S.-H. Park, O. Simeone, O. Sahin, and S. Shamai, "Robust and efficient distributed compression for cloud radio access networks," IEEE Trans. Veh. Commun., vol. 62, no. 2, pp. 692-703, Feb. 2013.

[7] Y. Zhou and W. Yu, "Optimized backhaul compression for uplink cloud radio access network," IEEE J. Sel. Areas Commun., to appear 2014.

[8] — , "Approximate bounds for limited backhaul uplink multicell processing with single-user compression," in Proc. IEEE CWIT, Jun. 2013, pp. 113-116.

[9] S. S. Christensen, R. Agarwal, E. Carvalho, and J. M. Cioffi, "Weighted sum-rate maximization using weighted MMSE for MIMO-BC beamforming design," IEEE Trans. Wireless Commun., vol. 7, no. 12, pp. 47924799, Jul. 2008.

[10] Q. Shi, M. Razaviyayn, Z.-Q. Luo, and C. He, "An iteratively weighted MMSE approach to distributed sum-utility maximization for a MIMO interfering broadcast channel," IEEE Trans. Signal Process., vol. 59, no. 9, pp. 4331-4340, Sep. 2011.

[11] C. T. K. Ng and H. Huang, "Linear precoding in cooperative MIMO cellular networks with limited coordination clusters," IEEE J. Sel. Areas Commun., vol. 28, no. 9, pp. 1446-1454, Sep. 2010.

[12] S.-H. Park, O. Simeone, O. Sahin, and S. Shamai, "Joint precoding and multivariate backhaul compression for the downlink of cloud radio access networks," IEEE Trans. Signal Process., vol. 61, no. 22, pp. 5646-5658, Nov. 2013.

[13] W. Yu, W. Rhee, S. Boyd, and J. M. Cioffi, "Iterative water-filling for Gaussian vector multiple-access channels," IEEE Trans. Inf. Theory, vol. 50, no. 1, pp. 145-152, 2004. 